\title{
NEOWISE-R OBSERVATION OF THE COOLEST KNOWN BROWN DWARF
}

\author{
Edward L. Wright ${ }^{1}$, Amy Mainzer ${ }^{2}$, J. Davy KirkPatrick ${ }^{3}$, Frank Masci $^{3}$, Michael C. Cushing ${ }^{4}$ James Bauer $^{2}$, \\ Sergio Fajardo-Acosta ${ }^{3}$, Christopher R. Gelino ${ }^{3}$, Charles A. Beichman ${ }^{3,5}$, M. F. Skrutskie ${ }^{6}$, T. Grav ${ }^{7}$, \\ Peter R. M. EISENHARdT ${ }^{2}$, AND Roc CUTRI ${ }^{3}$ \\ ${ }^{1}$ UCLA Astronomy, P.O. Box 951547, Los Angeles, CA 90095-1547, USA; wright@ astro.ucla.edu \\ 2 Jet Propulsion Laboratory, 4800 Oak Grove Drive, Pasadena, CA 91109, USA \\ ${ }^{3}$ Infrared Processing and Analysis Center, California Institute of Technology, Pasadena, CA 91125, USA \\ ${ }^{4}$ Department of Physics and Astronomy, University of Toledo, 2801 West Bancroft Street, Toledo, OH 43606-3328, USA \\ ${ }^{5}$ NASA Exoplanet Science Institute, MS 100-22, California Institute of Technology, Pasadena, CA 91125, USA \\ ${ }^{6}$ Department of Astronomy, University of Virginia, Charlottesville, VA 22904, USA \\ ${ }^{7}$ Planetary Science Institute, Tucson, AZ 85719, USA \\ Received 2014 May 27; accepted 2014 July 21; published 2014 September 26
}

\begin{abstract}
The Wide-field Infrared Survey Explorer (WISE) spacecraft has been reactivated as NEOWISE-R to characterize and search for near-Earth objects. The brown dwarf WISE J085510.83-071442.5 has now been re-observed by NEOWISE-R, and we confirm the results of Luhman, who found a very low effective temperature $(\approx 250 \mathrm{~K})$, a very high proper motion $\left(8^{\prime \prime} \cdot 1 \pm 00^{\prime} 1 \mathrm{yr}^{-1}\right)$, and a large parallax (454 \pm 45 mas). The large proper motion has separated the brown dwarf from the background sources that influenced the 2010 WISE data, allowing a measurement of a very red WISE color of $W 1-W 2>3.9 \mathrm{mag}$. A re-analysis of the 2010 WISE astrometry using only the W2 band, combined with the new NEOWISE-R 2014 position, gives an improved parallax of $448 \pm 33$ mas and a proper motion of 8."08 $\pm 00^{\prime \prime} 05 \mathrm{yr}^{-1}$. These are all consistent with values from Luhman.
\end{abstract}

Key words: astrometry - brown dwarfs - infrared: stars - solar neighborhood stars: individual (WISE J085510.83-071442.5) - stars: low-mass

Online-only material: color figures

\section{INTRODUCTION}

The Wide-field Infrared Survey Explorer (WISE; Wright et al. 2010) observed the entire sky in four infrared bands at 3.4, 4.6, 12 , and $22 \mu \mathrm{m}$ in early 2010 , then continued to observe in the 3.4 and $4.6 \mu \mathrm{m}$ bands until 2011 February. In 2011 February the spacecraft went into hibernation. In late 2012 a brief test showed that the spacecraft was still functional, and in 2013 the planetary science division of NASA funded a reactivation of the spacecraft to characterize and search for near-Earth objects, with Amy Mainzer as Principal Investigator of this new NEO WISE Reactivation (NEOWISE-R) mission. After passively cooling back to $73 \mathrm{~K}$, surveying the sky began again in 2013 December (Mainzer et al. 2014).

Both Luhman (2014a) and Kirkpatrick et al. (2014) noted that the source WISE J085510.83-071442.5 (also known as WISEA J085510.74-071442.5, hereafter W0855) had a large motion between the two epochs of WISE data in 2010 May and 2010 November, but since the source could not be seen in the Two Micron All Sky Survey (Skrutskie et al. 2006), this motion was unconfirmed. Luhman (2014b) also failed to see W0855 in the $J$-band images from the VISTA survey (McMahon et al. 2013), but was able to obtain data using the short wavelength bands of the Infrared Array Camera (Fazio et al. 2004) on the Spitzer Space Telescope (Werner et al. 2004) that confirmed the large motion of W0855 and showed a large parallax for this object as well. In addition, these data showed that W0855 was an extremely red source and that a clump of background sources had influenced the WISE photometry and astrometry taken in 2010. The low luminosity and red color of W0855 require effective temperatures near $250 \mathrm{~K}$ and masses well below the deuterium burning limit: 3-10 Jupiter masses for ages of 1-10 Gyr (Luhman 2014b). Further observations of W0855 and further searches for similar objects will aid our understanding of the relation between the population of more massive brown dwarfs and the population of exoplanets, both bound and freefloating (Sumi et al. 2011).

In 2014 May NEOWISE-R scanned over W0855 again, giving new unconfused position and photometry data for both W0855 and the clump of background sources. In this paper we use these data to derive an improved proper motion and parallax fit for W0855 and to derive uncontaminated colors in the WISE bands. Figure 1 shows postage stamps of the WISE W1 and W2 coadds for the three epochs. In addition we report an unsuccessful attempt to detect W0855 in the $H$ band.

\section{OBSERVATIONS}

The 2014 NEOWISE-R data are reported in Tables 1 and 2. These values were all obtained using the AllWISE profile fit photometry and astrometry software applied to the sets of frames containing W0855 in each epoch. The combined flux from W0855 and the background source clump gives a $W 2$ magnitude of $13.757 \pm 0.050$, which is reasonably close to the $13.633 \pm 0.038$ observed in 2010 May. All magnitudes and colors in this paper are reported on the Vega system. The $W 1-W 2$ color observed in 2010 was clearly contaminated by the background source clump. The new color $W 1-W 2=$ $3.803 \pm 0.329$ mag is extremely red, placing W0855 squarely in the region of Y dwarfs (Kirkpatrick et al. 2012). The signalto-noise ratio $(\mathrm{S} / \mathrm{N})$ in $W 1$ is quite low, so the limits on the color are asymmetric: at $2 \sigma$, the range of colors consistent with the data is 3.3-4.8 mag. Examination of Figure 1 shows that the NEOWISE-R $W 1$ flux may still be confused by a much fainter background source, and that the density of faint background 


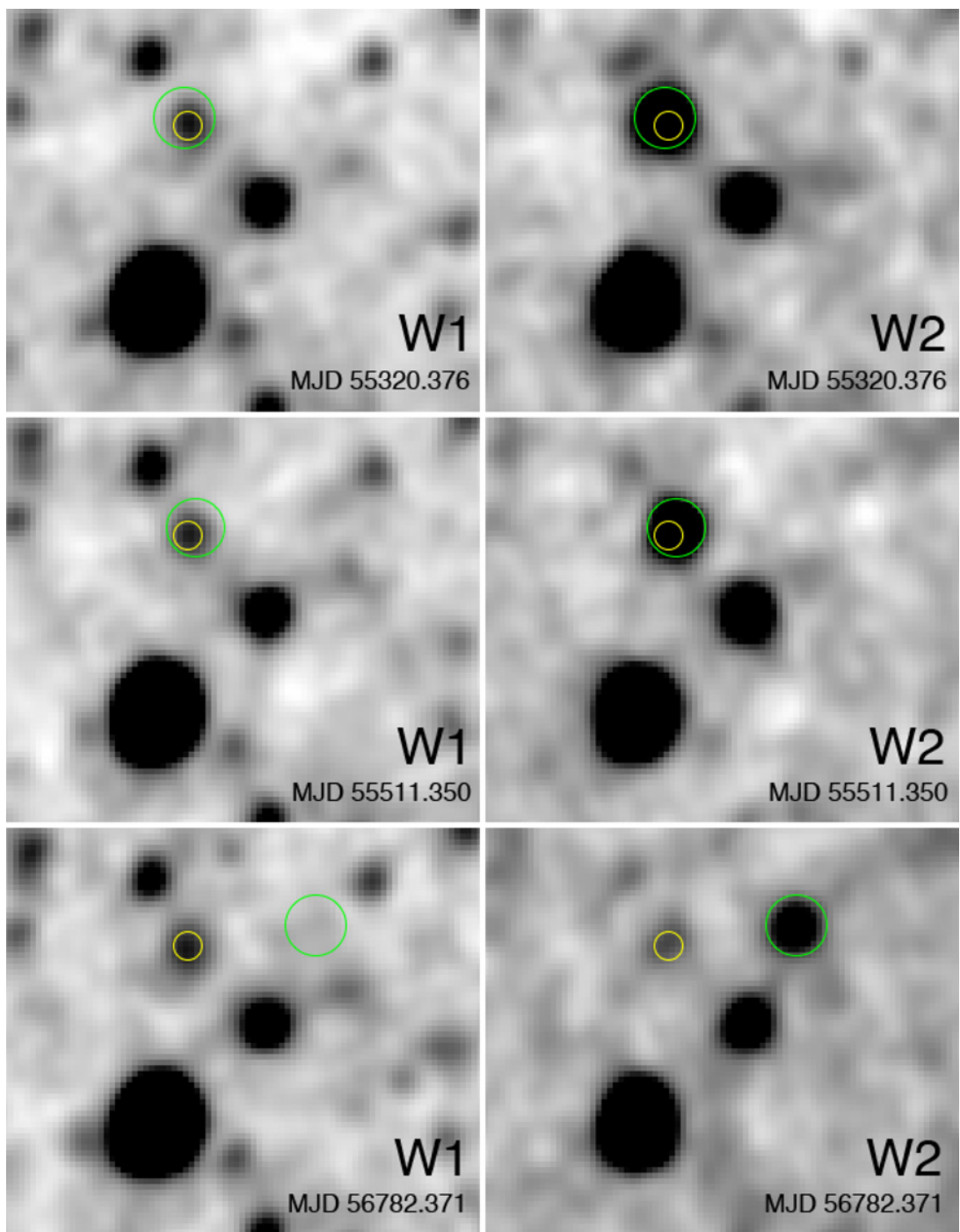

Figure 1. $W 1$ and $W 2$ images of the W0855 field for the three WISE epochs. The small yellow circle marks the background source clump. The larger green circle marks the WISE position for each epoch. Each panel is $100^{\prime \prime}$ wide, with north at the top and east on the left.

(A color version of this figure is available in the online journal.)

Table 1

New Astrometric Data [J2000]

\begin{tabular}{lccccc}
\hline \hline MJD & $\alpha\left(^{\circ}\right)$ & $\begin{array}{c}\sigma_{\alpha} \\
(\mathrm{mas})\end{array}$ & $\delta\left(^{\circ}\right)$ & $\begin{array}{c}\sigma_{\delta} \\
(\mathrm{mas})\end{array}$ & Notes \\
\hline 55320.376 & 133.7951649 & 101 & -7.2451021 & 111 & WISE W2 only \\
55511.350 & 133.7944072 & 109 & -7.2450719 & 120 & WISE W2 only \\
56782.371 & 133.7862181 & 158 & -7.2442562 & 175 & NEOWISE-R \\
\hline 56782.371 & 133.7949499 & 207 & -7.2455978 & 231 & Bkgnd, W1 and W2 \\
56782.371 & 133.7949398 & 486 & -7.2452772 & 556 & Bkgnd, W2 only \\
\hline
\end{tabular}

objects is such that the $W 1$ flux measurement of W0855 is confusion limited.

In order to reduce the effects of confusion, difference images of the 2014 May co-add minus the 2010 May co-add were constructed. These are shown in Figure 2. Analysis of these images shows that the $W 1$ flux of W0855 is $7.5 \pm 6.5 \mu \mathrm{Jy}$. This is about $2.3 \sigma$ less than the value found by the standard profile fit photometry, indicating that the 2014 May epoch probably still suffers from confusing background sources. The central value
Table 2

New Photometric Observations

\begin{tabular}{lcc}
\hline \hline Filter & Magnitude & Notes \\
\hline$W 1$ & $16.117 \pm 0.073$ & Background clump \\
$W 2$ & $15.441 \pm 0.152$ & Background clump \\
\hline$H$ & $>22.7$ & W0855 \\
$W 1$ & $17.819 \pm 0.327$ & W0855 \\
$W 2$ & $14.016 \pm 0.048$ & W0855 \\
\hline
\end{tabular}

of the $W 1-W 2$ color is $5.0 \mathrm{mag}$, with a $2 \sigma$ lower limit on the color of 3.9 mag. W0855 is an extremely red source.

W0855 was observed on 2014 February 9 in the $H(1.6 \mu \mathrm{m})$ band with NIRC2 behind the Keck II LGS-AO system (Wizinowich et al. 2006; van Dam et al. 2006), but the 40" field of view was improperly centered due to the large proper motion of W0855. W0855 was re-observed on 2014 May 18 but no detection was made in 30 minutes of integration, and a $3 \sigma$ upper limit is reported in Table 2. This upper limit shows that W0855 is redder than W1828+2650, one of the reddest known brown dwarfs, in the $H-W 2$ color (Kirkpatrick et al. 2012). 


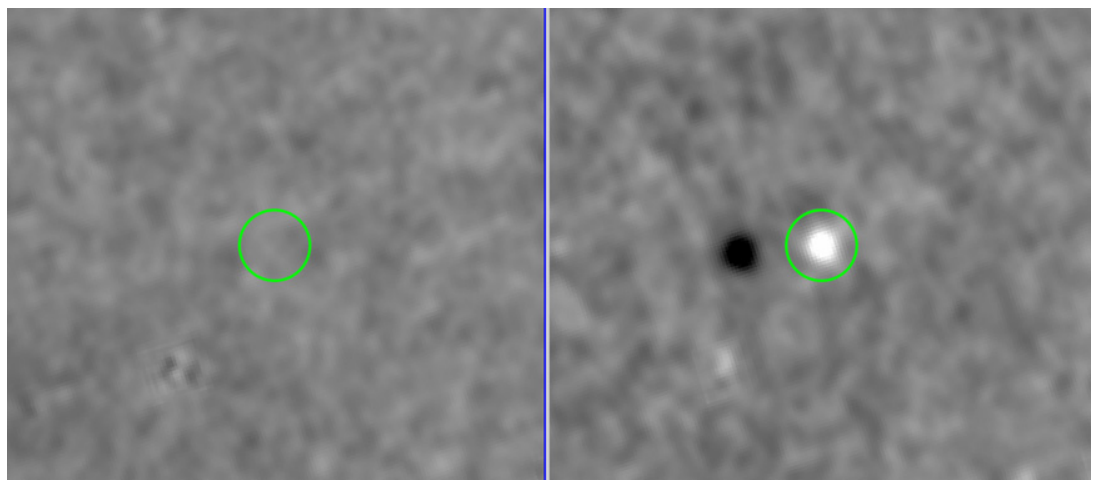

Figure 2. 2014 May minus 2010 May images. Left panel: W1. Right panel: W2. Width of each image is 3'5. The circle marks the 2014 May position of W0855. (A color version of this figure is available in the online journal.)

The upper limit $J>23$ reported by Luhman (2014b) shows that W0855 is also nearly as red or redder than W1828+2650 in the $J-W 2$ color.

\section{ASTROMETRIC FIT}

Also reported in Table 1 are new analyses of the 2010 WISE data. For these positions, only the $W 2$ data were used. Since the detection of W0855 is dominated by $W 2$ and the detection of the background source clump is dominated by $W 1$, the positions will be less affected by confusion.

Even with the positions derived from $W 2$ data alone, we expect to see an astrometric shift due to the influence of the background source clump. Luhman (2014b) went to considerable lengths to correct for this influence. In order to check Luhman's analysis using an independent method, we use a fairly simple analysis that assumes that the true position is related to the observed position by

$$
(\alpha, \delta)_{\mathrm{true}}=f \times(\alpha, \delta)_{\mathrm{obs}}+(1-f) \times(\alpha, \delta)_{\mathrm{bkgnd}}
$$

We model the true position using the usual five parameters: $\Delta \alpha_{\circ}, \Delta \delta_{\circ}, \mu_{\alpha}=\cos \delta d \alpha / d t, \mu_{\delta}=d \delta / d t$, and parallax $\varpi$. This introduces a sixth parameter, $f$, into the analysis in addition to the usual five parameters. Only the first two observations by WISE are affected by this new parameter.

We end up with the standard equations (Marsh et al. 2013) for $i=3-5$ :

$$
\begin{aligned}
& x_{i}=\cos \delta_{1}\left(\alpha_{i}-\alpha_{\mathrm{bkgnd}}\right)=\Delta \alpha+\mu_{\alpha}\left(t_{i}-t_{1}\right)+\varpi \boldsymbol{R}_{i} \cdot \hat{W}, \\
& y_{i}=\delta_{i}-\delta_{\mathrm{bkgnd}}=\Delta \delta+\mu_{\delta}\left(t_{i}-t_{1}\right)-\varpi \boldsymbol{R}_{i} \cdot \hat{N}
\end{aligned}
$$

and modified equations for $i=1$ and 2 :

$$
\begin{aligned}
x_{i}= & \cos \delta_{1}\left(\alpha_{i}-\alpha_{\text {bkgnd }}\right)=\Delta \alpha+\mu_{\alpha}\left(t_{i}-t_{1}\right) \\
& +\varpi \boldsymbol{R}_{i} \cdot \hat{W}-(f-1) x_{i}, \\
y_{i}= & \delta_{i}-\delta_{\text {bkgnd }}=\Delta \delta+\mu_{\delta}\left(t_{i}-t_{1}\right)-\varpi \boldsymbol{R}_{i} \cdot \hat{N}-(f-1) y_{i},
\end{aligned}
$$

where $t_{i}$ is the observation time (yr) of the $i$ th astrometric measurement and $R_{i}$ is the vector position of the observer relative to the Sun in celestial coordinates and astronomical units. $\hat{N}$ and $\hat{W}$ are unit vectors pointing north and west from the position of the source. Epochs 3 and 4 are the Spitzer positions reported by Luhman (2014b). $R_{i}$ is the position of the Earth for
WISE observations; for Spitzer observations, $R_{i}$ is the position of the spacecraft. The observed positional difference on the lefthand side is in arcseconds, the parameters $\Delta \alpha$ and $\Delta \delta$ are in arcseconds, the proper motion $\mu_{\alpha}$ and $\mu_{\delta}$ are in $\operatorname{arcsec} \mathrm{yr}^{-1}$, and the parallax $\varpi$ is in arcseconds.

In 2010, $x_{i}$ and $y_{i}$ are small and the value for the parameter $f$ is close to one, so the non-standard terms $(f-1) x_{i}$ and $(f-1) y_{i}$ on the right-hand side of Equation (3) do not cause problems. They do, however, require inflating the standard deviations of the 2010 data points by a factor of $f$ since a change of $x$ by $f \sigma$ is necessary to change the left-hand side of Equation (3) by $\sigma$. An iterative cycle of fitting for $f$, reweighting, then refitting for $f$ converges rapidly. In addition to this error inflation, there is an error with the form $(f-1) \sigma_{\text {bkgnd }}$ which is perfectly correlated across all the 2010 data. The background clump is faint in $W 2$ leading to an imprecise position, but the $W 2$-only position is not affected by color differences among the clump members. The effects due to the uncertainty in the clump position, the $(f-1) \sigma_{\text {bkgnd }}$ term, are significant and need to be added in quadrature to the errors from the least-squares fit. This was done by running the solutions for three background clump positions: the nominal position, a position with $+1 \sigma_{\delta}$, and a position with $+1 \sigma_{\alpha}$. Differences between these solutions give the sensitivity of the parameters to the background clump position errors. The final uncertainty on a parameter, such as $\varpi$, is given by $\sigma(\varpi)^{2}=$ $\sigma(\mathrm{LSQ})^{2}+\left(\sigma\left(\delta_{\text {bkgnd }}\right) \partial \varpi / \partial \delta_{\text {bkgnd }}\right)^{2}+\left(\sigma\left(\alpha_{\text {bkgnd }}\right) \partial \varpi / \partial \alpha_{\text {bkgnd }}\right)^{2}$, where $\sigma(\mathrm{LSQ})$ is the usual error reported by the leastsquares fit. The resulting parameter values and uncertainties are $\mu_{\alpha}=-8^{\prime \prime} .051 \pm 0.047 \mathrm{yr}^{-1}, \mu_{\delta}=0.657 \pm 0.050 \mathrm{yr}^{-1}$, parallax $=448 \pm 33$ mas, and $f=1.237 \pm 0.071$. For this fit $\chi^{2}=2.59$ with 4 degrees of freedom. Figure 3 shows the data and the fit with the proper motion removed.

W0855 passed from east to west of the background clump during 2010, and the mean right ascension during 2010 is well determined. As a result, our $\mu_{\alpha}$ differs from Luhman's value by

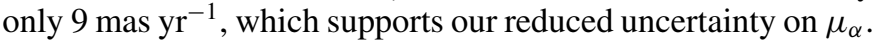
The proper motion in right ascension is highly correlated with the parallax, so by reducing the uncertainty in $\mu_{\alpha}$ we also get a reduced uncertainty in the parallax. The difference between this paper and Luhman (2014b) in $\mu_{\delta}$ is $43 \pm 86$ mas yr$^{-1}$ when we use the $W 2$-only position for the clump of background sources.

The value of $f$ implies that the centroid is a weighted sum of W0855 and the background source clump with weights $1 / f=81 \% \pm 4 \%$ for $\mathrm{W} 0855$ and $19 \% \mp 4 \%$ for the background source clump. The total $W 2$ flux is divided with $79 \% \pm 2.5 \%$ from W0855 and $21 \% \mp 2.5 \%$ from the background source clump. Thus the derived value for $f$ is reasonable. 


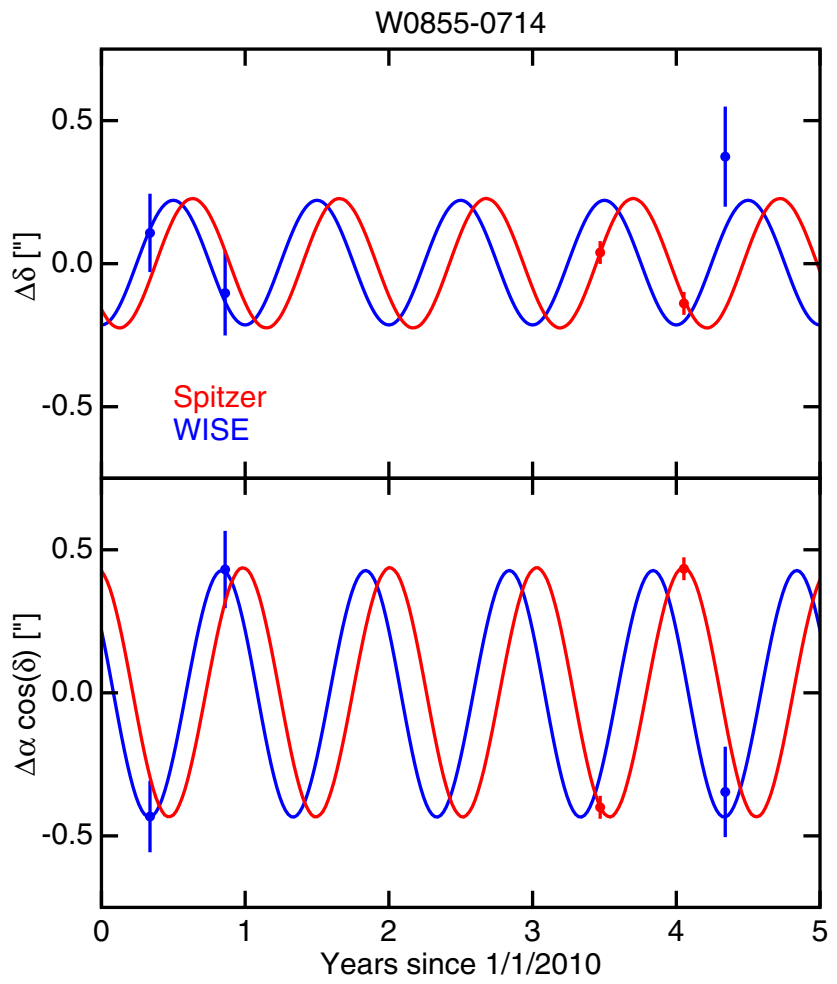

Figure 3. Astrometric data and fit for W0855. The constant and proper motion terms have been taken out in order to clearly show the parallactic motion. The parallax curve for Spitzer has a different phase due to its Earth-trailing orbit.

(A color version of this figure is available in the online journal.)

Our conclusion about the astrometry of W0855 is that Luhman's procedure for correcting the 2010 data for the effect of the background source clump was quite successful, and our new data and independent analysis technique give a parallax only 6 mas different than that of Luhman (2014b). Further astrometry of W0855 could reduce our reliance on the confused WISE 2010 data and this may come from NEOWISE-R, which should get five more epochs, from further Spitzer observations, or from Hubble Space Telescope (HST) detections of the J- or $H$-band flux.

\section{DISCUSSION}

The $\geqslant \mathrm{Y} 2$ brown dwarf $\mathrm{W} 1828+2650$ is so faint at $J$ and $H$ that the HST is needed to measure an accurate color: $F 140 W-W 2=8.76 \pm 0.11 \mathrm{mag}$ for this very red object, but W1828+2650 is not of extremely low luminosity given its absolute magnitude $M_{W 2}=14.17 \pm 0.25$ (Beichman et al. 2013). The distant white dwarf companion WD0806-661B has $F 125 W-[4.5]=8.81 \pm 0.14 \mathrm{mag}$ (C. Gelino et al. 2014, in preparation; Luhman et al. 2012) but an absolute magnitude of $M_{4.5}=15.46 \pm 0.07$ (Luhman et al. 2012). On the other hand, the Y1 brown dwarf W0350-5658 is bluer, with $F 140 W-$ $W 2=7.57 \pm 0.21 \mathrm{mag}$, but much less luminous with $M_{W 2}=$ $17.05 \pm 0.38$ (Marsh et al. 2013). Thus W0855, with $M_{W 2}=$ $17.27 \pm 0.17$, is less luminous than the least luminous previously known brown dwarfs, and as red or redder than the reddest previously known brown dwarfs. Many more objects redder than W0855 or less luminous than W0855 are needed to understand the trend and scatter of the luminosity versus color relation for these very low luminosity objects.

We are fortunate that W0855 is approximately two magnitudes above the AllWISE catalog limit, so a full search of the entire catalog would cover a volume $\left(10^{0.8}\right)^{1.5}=15.85$ larger than the volume of the sphere containing W0855. Thus there is a good possibility that many more examples of W0855-like objects exist in the AllWISE catalog, but in the absence of a third epoch to confirm the existence of a moving W2-only source it is not practical to obtain followup data from Spitzer or any ground-based telescope. The "statistics of one" limit the precision of any estimated number, but Kerman (2011) recommends using a $\lambda^{-2 / 3}$ prior for the Poisson rate $\lambda$, which gives a posterior probability density $\propto \lambda^{1 / 3} \exp (-\lambda)$ when $n=1$ objects have been seen. This posterior has its 16 th percentile at 0.33 , its median at $n=1.01$, and its 84 th percentile at 2.33. With this posterior, we estimate the number of objects that a survey of 15.85 times more volume would see, again using a Poisson distribution. This calculation gives a 16-84th percentile range of 4-35, with a median of 15 .

The combination of NEOWISE-R data with the AllWISE database will provide the additional observation epochs needed to confirm AllWISE motion detections of low $\mathrm{S} / \mathrm{N}$ sources, making it practical to find more W0855-like objects if they exist.

\section{CONCLUSION}

Early data from NEOWISE-R have provided new astrometric and photometric parameters for W0855. These are all consistent with the results of Luhman (2014b) and confirm that W0855 is an extremely red object with an extremely faint absolute magnitude $M_{W 2}=17.27 \pm 0.17$. W0855 shows that a population of very cold brown dwarfs exists that are so red that they are effectively unobservable from the ground at near-IR wavelengths. Since W0855 is two magnitudes brighter than the detection limit of WISE in the W2 band, an astrometric analysis of all the NEOWISE-R data down to the catalog limit could lead to the discovery of 4-35 similar objects.

This publication makes use of data products from the Widefield Infrared Survey Explorer, which is a joint project of the University of California, Los Angeles, and the Jet Propulsion Laboratory/California Institute of Technology, funded by the National Aeronautics and Space Administration. This publication makes use of data products from NEOWISE, which is a project of the Jet Propulsion Laboratory/California Institute of Technology, funded by the National Aeronautics and Space Administration.

Facilities: WISE, Keck:II (NIRC2-LGSAO)

\section{REFERENCES}

Beichman, C., Gelino, C. R., Kirkpatrick, J. D., et al. 2013, ApJ, 764, 101 Fazio, G. G., Hora, J. L., Allen, L. E., et al. 2004, ApJS, 154, 10 Kerman, J. 2011, EJSta, 5, 1450

Kirkpatrick, J. D., Gelino, C. R., Cushing, M. C., et al. 2012, ApJ, 753, 156 Kirkpatrick, J. D., Schneider, A., Fajardo-Acosta, S., et al. 2014, ApJ, 783, 122 Luhman, K. L. 2014a, ApJ, 781, 4

Luhman, K. L. 2014b, ApJL, 786, L18

Luhman, K. L., Burgasser, A. J., Labbé, I., et al. 2012, ApJ, 744, 135

Mainzer, A., Bauer, J., Cutri, R., et al. 2014, ApJ, 792, 30

Marsh, K. A., Wright, E. L., Kirkpatrick, J. D., et al. 2013, ApJ, 762, 119

McMahon, R. G., Banerji, M., Gonzalez, E., et al. 2013, Msngr, 154, 35

Skrutskie, M. F., Cutri, R. M., Stiening, R., et al. 2006, AJ, 131, 1163

Sumi, T., Kamiya, K., Bennett, D. P., et al. 2011, Natur, 473, 349

van Dam, M. A., Bouchez, A. H., Le Mignant, D., et al. 2006, PASP, 118, 310 Werner, M. W., Roellig, T. L., Low, F. J., et al. 2004, ApJS, 154, 1

Wizinowich, P. L., Le Mignant, D., Bouchez, A. H., et al. 2006, PASP, 118, 297 Wright, E. L., Eisenhardt, P. R. M., Mainzer, A. K., et al. 2010, AJ, 140, 1868 